\title{
DESIGN AND CONTROL OF A SMART BED FOR PRESSURE ULCER PREVENTION
}

\author{
by \\ ZACHARY GOVIER BRUSH
}

\author{
Presented to the Faculty of the Graduate School of \\ The University of Texas at Arlington in Partial Fulfillment \\ of the Requirements \\ for the Degree of
}

MASTER OF SCIENCE IN MECHANICAL ENGINEERING

THE UNIVERSITY OF TEXAS AT ARLINGTON

May 2013 
Copyright @ by ZACHARY GOVIER BRUSH 2013

All Rights Reserved 
To my parents, Kevin and Ruth Brush, for supporting me throughout my education and motivating me to always strive for excellence. 


\section{ACKNOWLEDGEMENTS}

I would like to thank Dr. Alan Bowling, Dr. Panayiotis Shiakolas, and Dr. Mohammad Mayyas for reviewing my work as members of my thesis defense committee. I would like to thank Prof. Mehrdad Nourani in the Department of Electrical Engineering at the University of Texas at Dallas (UTD), and Prof. Deborah Behan in the College of Nursing at the University of Texas at Arlington (UTA), for their thoughts, expertise, and collaboration in the conceptual design of the Smartbed. I would also like to thank Dr. HongQing Tian for his assistance and expertise in writing the microcontroller code and wiring the servoamplifier and microcontroller connections. Finally, financial support for the preliminary work on the Smartbed was provided by the Collaborative Research Funding Program in Medical Technologies run by the UTA-UTD-Texas Instruments (TI)/Texas Health Research and Education (THRE) Institute Medical Technology Research Program, and this assistance is greatly appreciated.

April 19, 2013 


\title{
ABSTRACT \\ DESIGN AND CONTROL OF A SMART BED FOR PRESSURE ULCER PREVENTION
}

\author{
ZACHARY GOVIER BRUSH, M.S.
}

The University of Texas at Arlington, 2013

Supervising Professor: Alan Bowling

This work details the design, simulation, and experimental testing of a mechanically actuated smart hospital bed for the prevention of pressure ulcers in hospital patients. The smart hospital bed, or Smartbed, is designed to improve the "turning" process currently performed by health care workers, ensuring that patients are turned consistently and decreasing the labor requirement for caregivers. The mechanical structure of the bed is described, along with its advantages over current Smartbed products. Next, dynamic models of the Smartbed actuating systems are discussed along with descriptions of the devised single-unit and overall bed control systems. The control equations determined in simulation are then simplified, allowing them to be implemented in real time by a microcontroller. Finally, the simplified control system is tested against the original dynamic model in simulation, and the actuation of one constructed unit of the multi-unit bed platform is tested with open

loop user input, validating design choices and improvements made to the hardware of the mechanism. 


\section{TABLE OF CONTENTS}

ACKNOWLEDGEMENTS ............................. iv

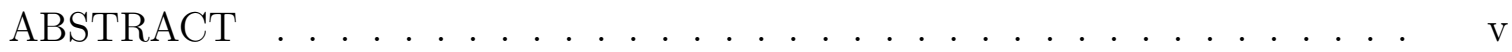

LIST OF ILLUSTRATIONS . . . . . . . . . . . . . . . . . . . . vii

Chapter Page

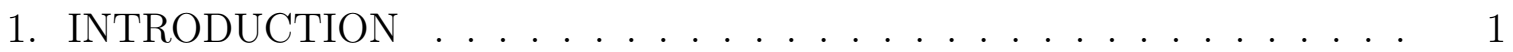

2. SMART BED DESIGN . . . . . . . . . . . . . . . . . 4

2.1 Overall Bed Design Concept . . . . . . . . . . . . . . 4

2.2 Individual Tile Design _ . . . . . . . . . . . . . . . . . . . . 7

3. DYNAMIC MODELING AND CONTROL . . . . . . . . . . . . . . . 11

3.1 Dynamic Modeling of an Individual Tile . . . . . . . . . . . . . . . . 11

3.2 Control System Equations . . . . . . . . . . . . . . . . 12

4. CONTROL SYSTEM APPLICATION . . . . . . . . . . . . 18

4.1 Simplification of the Control Equations . . . . . . . . . . . . . . . 19

4.2 Consideration of Actuator Dynamics . . . . . . . . . . . . 23

5. RESULTS AND EXPERIMENTAL TESTING . . . . . . . . . . . 26

5.1 Control Program . . . . . . . . . . . . . . . . 26

5.2 Mechanism Hardware . . . . . . . . . . . . . . . . . . . . . . . . . 29

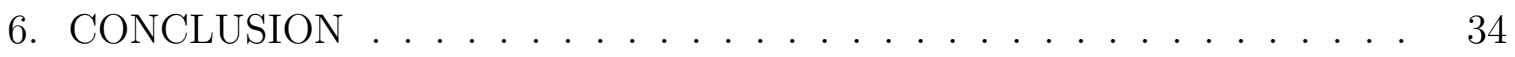
Appendix

A. EQUIPMENT LIST . . . . . . . . . . . . . . . . . . . 36

REFERENCES . . . . . . . . . . . . . . . . . . . 38

BIOGRAPHICAL STATEMENT . . . . . . . . . . . . . . . . . . . . 41 


\section{LIST OF ILLUSTRATIONS}

Figure $\quad$ Page

2.1 Soft, non-grasp manipulation . . . . . . . . . . . . . . . 5

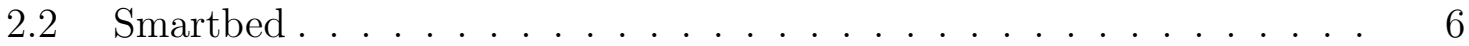

2.3 One tile of the Smartbed . . . . . . . . . . . . . . 7

2.4 Structure of the top joints and connections . . . . . . . . . . . . 8

3.1 Generalized coordinates of the mechanism. An additional pair of coordinates for each leg and one representing the rotation of the plate body with respect to one of the joint frames are not shown . . . . . . . . . . 12

3.2 Operational space velocities of the mechanism: vertical translation, $\overrightarrow{\boldsymbol{h}}_{\mathbf{3}}$, and two axes of rotation, $\overrightarrow{\boldsymbol{\theta}}_{\mathbf{1}}$ and $\overrightarrow{\boldsymbol{\theta}}_{\mathbf{2}} \ldots \ldots \ldots 14$

3.3 Top view of the mechanism schematic, illustrating the definitions of $l_{1}$, $l_{4}$, and $l_{5}$ with respect to the threaded rods $r_{1}, r_{2}$, and $r_{3} \ldots 16$

4.1 Illustration of the simplified mechanism model, relating the operational space variables $\theta_{1}, \theta_{2}$, and $h_{3}$ and the joint space variables, $q_{1}, q_{2}$, and $q_{3}$. The front of the mechanism in each image is labeled for reference . 19

4.2 Error in approximated $\theta_{1}$ value over the full operational domain of $\theta_{1}$. The solid line at the bottom represents where there is exactly zero error

4.3 a) Error in approximated $\theta_{2}$ value over the full operational domain of $\theta_{2}$. b) Error in approximated $h_{3}\left(h_{m i d}\right)$ value over the full operational domain of $h_{3}$. The blue lines represent the ideal case where there is exactly zero error . . . . . . . . . . . . . . . . 
4.4 Inclined plane analogous to the incline of the leadscrew's thread. $l$ is the lead length (the vertical distance traveled when the thread completes one rotation) and $\pi d_{m}$ is the mean circumference of the thread for one

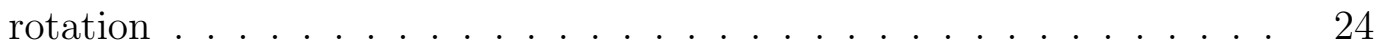

5.1 Control diagram for the mechanical actuators in each tile . . . . . . 26

5.2 Desired trapezoidal trajectories shown as dotted lines are followed closely by the actual mechanism positions (a) and velocities (b) in sim-

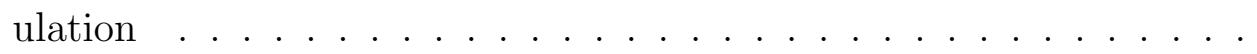

5.3 Motor torques required to create the previously illustrated operational space trajectories. The spikes represent where the discontinuities in desired acceleration exist at $t(0), t_{\text {rise }}, t_{\text {fall }}$, and $t_{\text {total }}$. In addition, at $t \approx 18$, the sign of $\tau_{2}$ changing from + to - is apparent . . . . . . .

5.4 One mechanism, fully constructed. This image includes the new motors, shaft couplings, a hard plastic bracket, and all necessary electronics . .

5.5 Torque-speed curves shown against the working range of the motor. All curves are well within the thermal, power, and motor torque-speed limits 30

5.6 a) Motor, shaft coupling, worm, worm gear, and leadscrew. b) The offwhite bracket locks the leadscrew and threaded rods in place through a thick washer, a rotational bearing, a thin washer, and a rapid prototype connector stuck directly on the leadscrew nut. c) The 3-D model used to create the rapid-prototype parts . . . . . . . . . . . . . 31

5.7 Control diagram for the mechanical actuators in each tile . . . . . . . 32 


\section{CHAPTER 1}

\section{INTRODUCTION}

Pressure ulcers (PUs), also known as decubitus ulcers or bed sores, are localized areas of damage to skin and adjacent tissues due to applied pressure, friction, or shear. These sores most often occur over bony prominences such as the hips, heels, spine, and other joints and are the result of a prolonged lack of blood flow to the affected area. Although the development of these sores is based on a variety of factors such as age, nutrition, skin moisture, and general health, PUs are usually found in patients suffering from immobility, spinal cord injury, or other severe illnesses and the elderly [1], [2], [3].

PUs are not just a source of pain and discomfort for bed and chair bound patients - these sores often act as avenues for infection and other complications, some of which can lead to permanent wounds and loss of life [4]. Over time, these sores can cause the skin and underlying tissues to die, exposing muscle and bone underneath the sores [5]. The treatment of these ulcers can be expensive, with patients spending an estimated $\$ 4,000$ to $\$ 40,000$ in additional hospital costs and care depending on the severity [6]. Despite the effort of the scientific and medical communities to prevent these sores, an estimated $3 \%$ to $11 \%$ of hospital patients and up to $30 \%$ of spinal cord injury and elderly hip replacement patients develop them during their hospital stay $[4]-[7]$.

Currently, the most common practice to prevent PUs is for nurses or caregivers to physically turn patients over from side to side approximately every two hours. This manual repositioning allows parts of a patient's body to recover while the contact 
forces between their body and the bed are applied elsewhere. However, this system is flawed: according to [8], only about $66 \%$ of patients receive this treatment on a regular basis, most likely due to nursing labor shortages. Turning patients is also shown to be a significant cause of lower back pain in health care workers [9]. In addition the turning process creates distortion and shear stress in the skin, causing damage that this process is meant to prevent [10]. The deployment of hospital beds that could reposition patients consistently without creating shear on patient's skin would both improve the prevention of PUs and significantly reduce the time, labor, and cost of their treatment.

Hospital beds of this nature, often called smart beds, are already on the market and have been for some time. One common example is the alternating pressure air mattress (APAM), which rotates users side to side by inflating and deflating airfilled cells in the mattress. While these products show promise, their effectiveness is inconclusive [7], [11], [12], [13]. Three-piece mechanical beds that can incline an individual's back and legs have also been created, some of which include pressure sensor arrays to provide feedback and APAMs with limited turning capabilities [14], [15]. While these have been shown to reduce the prevalence of PUs, current models are unable to reposition a patient onto their side and remove pressure on their back without the assistance of a hospital worker.

The goal of this work is to improve current smart bed systems by replicating the patient turning processes solely through actuation of the bed without the need for a caregiver to exert themselves. The design of this bed is described in detail in chapter 2, especially with respect to PU prevention recommendations found in the literature. In chapter 3, components of the smart bed are modeled to illustrate their dynamic properties, and the control system for the bed is designed and simulated. Chapter 4 continues the design process, taking the theoretical control system developed in 
simulation, simplifying it, and making it practical to apply to a real mechanism. Finally, in chapter 5 the simplified model is tested in simulation, and the fabrication and experimental testing of one repeated unit of the mechanical bed is documented and discussed. 


\section{CHAPTER 2}

\section{SMART BED DESIGN}

The smart bed design is focused on providing cyclic pressure loading on patients' bodies to prevent the development of pressure ulcers. A general description of desired bed properties is stated first, followed by specific details of the actuating mechanisms and control system structure.

\subsection{Overall Bed Design Concept}

The smart hospital bed, Smartbed, proposed in this work improves upon the designs of currently available products by including an actuating mechanism capable of manipulating patients' bodies without being physically assisted by a caregiver. While this will eliminate the need for assistance in turning the patient, it is not meant to be utilized without a nurse's care and supervision - the Smartbed is a tool designed to remove one of the more time and labor intensive tasks of caregivers as opposed to replacing their presence all together.

According to [14] and [16], complete or nearly complete removal of pressure on every area of patients' bodies by periodic, cyclic loading is desirable if not necessary for the total prevention of PUs. Therefore, the Smartbed must be capable of redistributing the forces on a patient's body so that total off-loading of the forces on all areas of the body at different times can be accomplished. However, the goal is to perform this task without performing large movement of the patient that creates potentially damaging shear forces. In the Smartbed this is accomplished using a com- 
bination of repositioning and inflation/deflation of an attached APAM. This process is illustrated in Fig. 2.1.

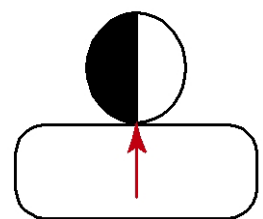

(a) High Pressure

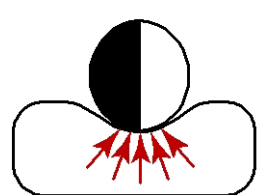

(b) Low Pressure

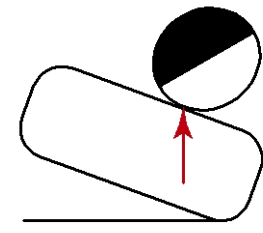

(c) High Pressure Tilt

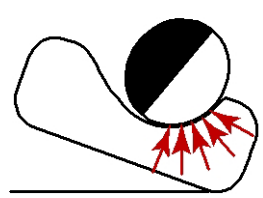

(d) Low Pressure Tilt

Figure 2.1. Soft, non-grasp manipulation.

In Fig. 2.1a, the mattress is highly pressurized. The body's weight is supported at a single point experiencing a large force, represented by the upward arrow. If the mattress is deflated, the surface deforms, allowing distribution and reduction of the force at each contact point, see Fig. 2.1b. However, the skin can stretch, increasing shear stress, as it sinks into the mattress. In addition, the contact point in Fig. 2.1a still experiences a normal force, and may not recover sufficiently.

Figure 2.1c shows repositioning using a stiff surface. The body rotates and the high force point moves across its surface. However, the force is still large and the body might move from the desired position, as occurs in manually turning the patient. Fig. 2.1d shows the smaller, redistributed forces resulting from simultaneous repositioning and deflating the mattress. The original contact point in Fig. 2.1a experiences no force, so the skin can recover. Movement of the body during rotation can reduce stretch and shear stress in the skin. The body is likely to remain in the final position and not rotate as far from its original position as in Fig. 2.1c. Overall, the body has not moved far from its original position, even though the mattress is tilted significantly. Periodically shifting the forces from one side to the other allows 
the skin on each side to recover, thereby achieving the effect of turning using small rotations.

As previously shown in Fig. 2.1, pneumatic and mechanical actuation deflates and tilts a flexible structure attached to a rigid one. These ideas can be extrapolated to obtain the preliminary tile design shown in Fig. 2.3, composed of a stiff parallel mechanism with an attached air bladder. The parallel mechanism has three degrees-of-freedom (DOFs). The air bladder has infinite DOFs, but only its internal pressure is controllable. This mechanism provides more controllable DOFs for force redistribution than either the air mattress or tilting/turning in Fig. 2.1 can alone.

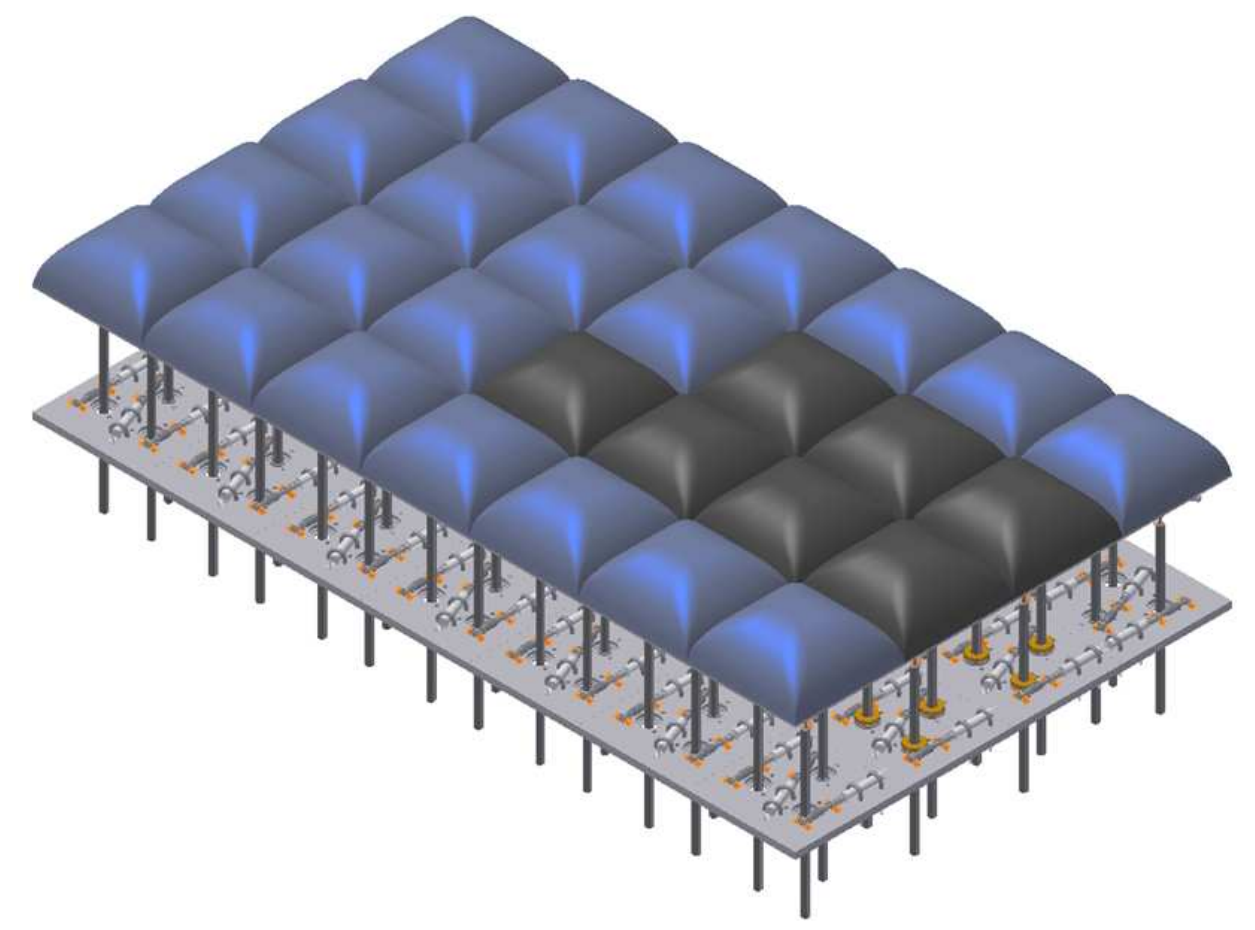

Figure 2.2. Smartbed.

Since the patient can be considered as several connected parts, it is reasonable to consider several tiles, as in Fig. 2.2, working independently or in concert to manip- 
ulate a patient. The proposed design includes two major aspects: a grid of mechanical plates actuated by motors that affect the shape of the bed and air bladders that act as variable pressure cushions. The grid of mechanical plates allows patients to be turned without the effort of a caregiver by providing vertical displacement and rotations to specific sections of the bed in a coordinated fashion. The air cushions, or bladders, can then redistribute local contact forces based on their level of inflation. This blend of mechanical and pneumatic actuation creates a surface with a high number of DOFs capable of performing complex manipulation of a human patient.

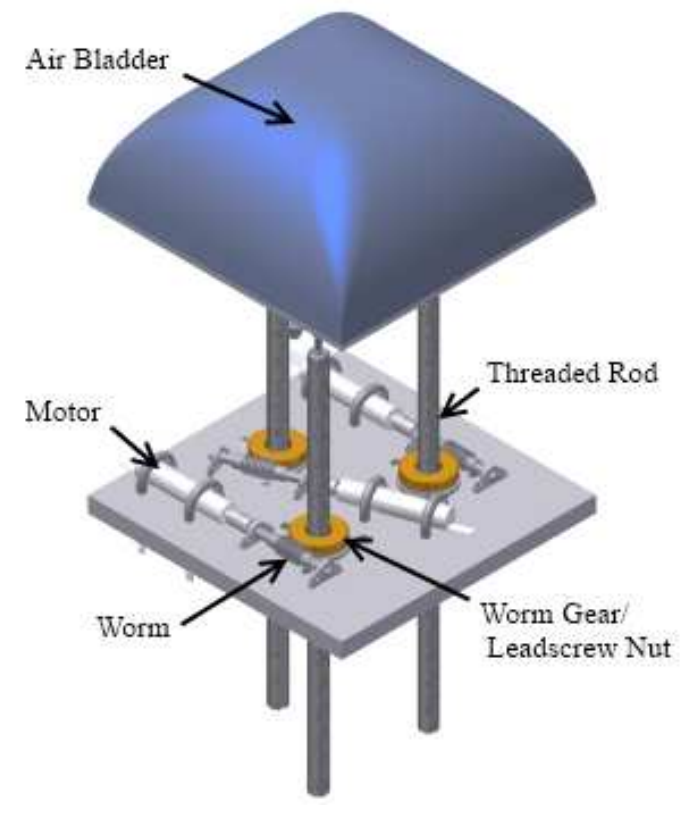

Figure 2.3. One tile of the Smartbed.

\subsection{Individual Tile Design}

The Smartbed is made up of a grid of plates, or tiles, each 0.3 meters by 0.3 meters ( 1 foot by 1 foot). The 28 tiles are arranged in a seven by four pattern, creating a bed that is 2.1 meters long and 1.2 meters wide. This is slightly longer 
and much wider than a standard hospital bed to accommodate larger patients, but a smaller size could be created as well.

Each tile is actuated by three 80 Watt, 24 Volt, brushless DC motors driven by three 50 Volt, 5 Ampere digital servoamplifiers (Fig. 2.3). Actuation from each motor to the plate is provided through a 50:1 speed ratio worm drive and a leadscrew connected to an Acme threaded rod with a lead of 4.2 millimeters per revolution $(1 / 6$ inch per revolution). In total, the gears create an overall gear ratio of 0.0847 millimeters per motor turn (0.00333 inches per motor turn). With a no load speed of 11,000 rpm, each threaded rod can raise and lower at a maximum speed of about 930 millimeters per minute (36.6 inches per minute). With an operating range of 381 millimeters (15 inches), the threaded rods can move from fully withdrawn to fully extended in approximately 30 seconds, including time for acceleration and deceleration.

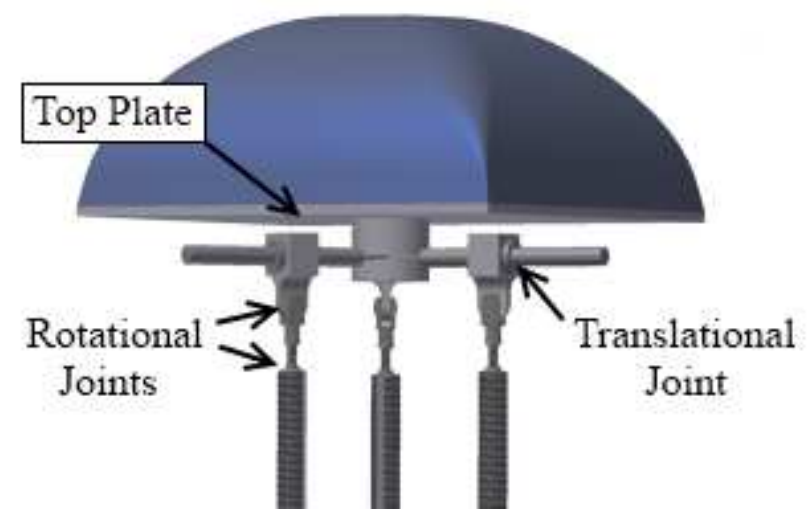

Figure 2.4. Structure of the top joints and connections.

The threaded rods are connected to the top plate through ball bearing rotational and translational joints at the top of each rod (Fig. 2.4). These in turn are connected to a rigid three-pronged structure mounted onto the bottom of the top plate with screws. This parallel mechanism has three degrees of freedom, defined 
in operational space as vertical translation and plate rotation about both horizontal axes. As designed on the prototype, the mechanism can create a $30^{\circ}$ angle across three tiles in either direction.

Electromechanical actuation was selected in order to create a sturdy, fixed bed structure without the need for power to remain static - incorporating a leadscrew with the previously described lead allows its static friction to provide more than adequate resistance to applied forces on top of the plate without motor actuation to prevent back-driving. Therefore, if the bed is disconnected from power, it would remain in its current position no matter what force is applied to it, assuming it doesn't fail. This form of actuation also moves smoothly because of the high gear ratio between the motor and rods, creating comfortable changes in bed positions. Finally, hydraulic systems can leak, which is undesirable in a clean, hospital setting.

The air bladder on top is included to distribute the force from high-risk areas for PUs to areas with less concentrated pressure. These acute areas are where bony prominences lie directly underneath skin tissue, including the heels, greater trochanters (hips), sacrum (lower spine), and coccyx (tailbone) [4]. PUs can also form on the gluteal muscles (buttocks), especially when the torso and head are elevated in semi-Fowler position.

Pressure distribution occurs due to the ability of the air cushions to deform over an infinite number of degrees of freedom. If kept at a constant air pressure, the cushion can withdraw under high pressure points, distributing the pressure to surrounding areas on the same tile. In addition, the pressure in each bladder is designed to be adjustable - air pumps provide air to each bladder individually. Combining this pneumatic actuation with a pressure sensor array on top of the bladders creates a force-based servo system by comparing and balancing the forces on all tiles. The design and control of the top sensors and air bladder are currently outside the scope of 
this research, although these aspects of the project were considered in the development of the mechanical hardware and control described in this work. 


\section{CHAPTER 3}

\section{DYNAMIC MODELING AND CONTROL}

In order to test the viability of this design and specify motor and servo amplifier specifications, a dynamic simulation of an individual unit of the Smartbed mechanism is conducted. This dynamic simulation is then used to determine control system equations using the computed-torque method and to size motors for the actual hardware.

\subsection{Dynamic Modeling of an Individual Tile}

A dynamic model of the mechanical system for each unit is created by dividing the parallel structure into 7 bodies: three threaded rods, three joint pieces, and the combined rigid top plate and three-pronged body (Fig. 3.1). The generalized coordinates $\boldsymbol{q}=\left[q_{1} \ldots q_{13}\right]^{T}$ are used to fully describe the structure's kinematics, and mass and inertia properties are added to each body according to estimations determined from solid models of each part.

The general form of the equations of motion for this multibody system is written as:

$$
M(\boldsymbol{q}) \ddot{\boldsymbol{q}}+\boldsymbol{b}(\dot{\boldsymbol{q}}, \boldsymbol{q})+\boldsymbol{g}(\boldsymbol{q})=\boldsymbol{\Gamma}(\dot{\boldsymbol{q}}, \boldsymbol{q})
$$

where $\dot{\boldsymbol{q}}$ and $\ddot{\boldsymbol{q}}$ represent each coordinate's generalized velocity and acceleration. The term $M(\boldsymbol{q}) \in \Re^{13 \times 13}$ is the mass matrix, $\boldsymbol{b}(\dot{\boldsymbol{q}}, \boldsymbol{q})$ includes the Coriolis and centrifugal terms, $\boldsymbol{g}(\boldsymbol{q})$ represents gravitational force, and $\boldsymbol{\Gamma}(\dot{\boldsymbol{q}}, \boldsymbol{q})$ denotes all other external forces acting on the mechanism. Since this is a three degree of freedom (DOF) system, kinematic constraints are used to relate the velocities of ten depen- 

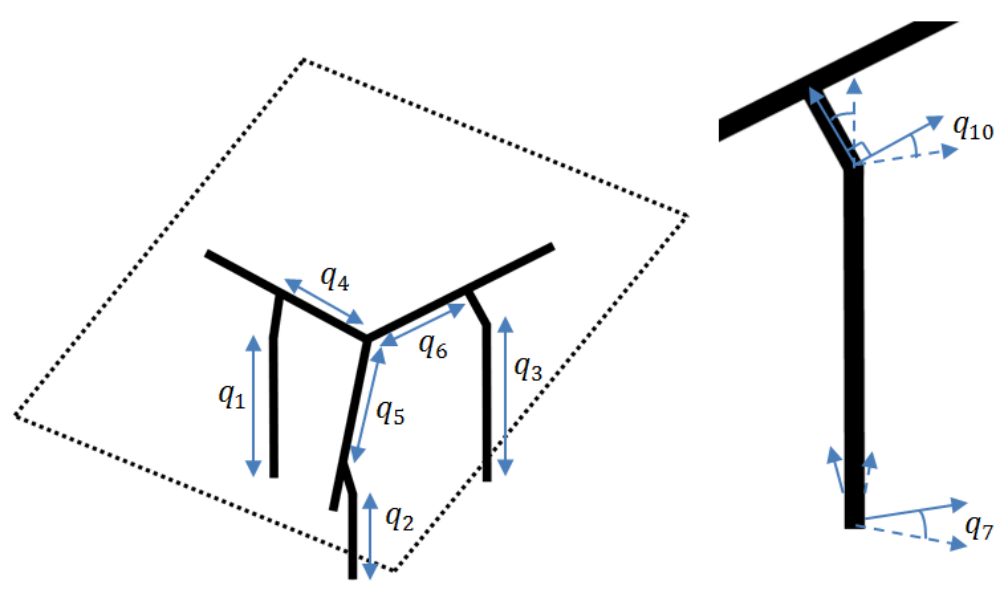

Figure 3.1. Generalized coordinates of the mechanism. An additional pair of coordinates for each leg and one representing the rotation of the plate body with respect to one of the joint frames are not shown.

dent coordinates to three independent coordinates (the extension of the three rods, $q_{1}, q_{2}$, and $\left.q_{3}\right)$. The analysis and modeling of these dynamic systems is based on Kane's method.

\subsection{Control System Equations}

The control system for the mechanism is developed using the computed-torque control method. Since an accurate model of the system dynamics is known because of the previously described dynamic modeling, the external forces, $\boldsymbol{\Gamma}(\dot{\boldsymbol{q}}, \boldsymbol{q})$, in $(3.1)$ are equivalent to the actuator torque from the three electric motors, as shown by:

$$
M(\boldsymbol{q}) \ddot{\boldsymbol{q}}+\boldsymbol{b}(\dot{\boldsymbol{q}}, \boldsymbol{q})+\boldsymbol{g}(\boldsymbol{q})=G^{T} \Upsilon(\dot{\boldsymbol{q}}, \boldsymbol{q})
$$

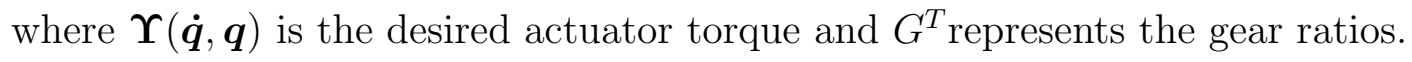
Using dynamic decoupling, also called feedback linearization, $\boldsymbol{\Gamma}(\dot{\boldsymbol{q}}, \boldsymbol{q})$ can then be written as:

$$
\Gamma(\dot{\boldsymbol{q}}, \boldsymbol{q})=\boldsymbol{\theta} \Gamma^{*}+\boldsymbol{\beta}
$$




$$
\begin{gathered}
\boldsymbol{\theta}=M(\boldsymbol{q}) \\
\boldsymbol{\beta}=\boldsymbol{b}(\dot{\boldsymbol{q}}, \boldsymbol{q})+\boldsymbol{g}(\boldsymbol{q})
\end{gathered}
$$

This assumes a unit mass system according to the definition $\Gamma^{*}=\boldsymbol{I} \ddot{\boldsymbol{q}}$. Now, the following control law with control constants $k_{p}$ and $k_{v}$ can be included:

$$
\Gamma^{*}(\dot{\boldsymbol{q}}, \boldsymbol{q})=\ddot{\boldsymbol{q}}_{\boldsymbol{d}}+k_{v}\left(\dot{\boldsymbol{q}}_{\boldsymbol{d}}-\dot{\boldsymbol{q}}\right)+k_{p}\left(\boldsymbol{q}_{\boldsymbol{d}}-\boldsymbol{q}\right)
$$

based on the desired trajectory over time for each of the three independent variables, $\boldsymbol{q}_{\boldsymbol{d}}, \dot{\boldsymbol{q}}_{\boldsymbol{d}}$, and $\ddot{\boldsymbol{q}}_{\boldsymbol{d}}$. As a result of the dynamic decoupling, $\boldsymbol{\Gamma}^{*}$ is now equal to the acceleration vector $\ddot{\boldsymbol{q}}$, resulting in the error equation in (3.5):

$$
\left(\ddot{\boldsymbol{q}}_{d}-\ddot{\boldsymbol{q}}\right)+k_{v}\left(\dot{\boldsymbol{q}}_{\boldsymbol{d}}-\dot{\boldsymbol{q}}\right)+k_{p}\left(\boldsymbol{q}_{\boldsymbol{d}}-\boldsymbol{q}\right)=\mathbf{0}
$$

Finally, by setting the desired operational space vectors as seen in (Fig. 3.2), a Jacobian can be introduced to create (3.8) and placed in (3.2) to determine the required actuator torques to follow the trajectory defined by $\boldsymbol{p}_{\boldsymbol{d}}, \boldsymbol{v}_{\boldsymbol{d}}$, and $\dot{\boldsymbol{v}}_{\boldsymbol{d}}$ in (3.9).

$$
\begin{aligned}
& \boldsymbol{v}=\left[\begin{array}{c}
v_{3} \\
\omega_{1} \\
\omega_{2}
\end{array}\right]=\boldsymbol{J} \dot{\boldsymbol{q}}, \\
& \dot{\boldsymbol{v}}=\dot{\boldsymbol{J}} \dot{\boldsymbol{q}}+\boldsymbol{J} \ddot{\boldsymbol{q}}, \\
& \ddot{\boldsymbol{q}}=\boldsymbol{J}^{-1}(\dot{\boldsymbol{v}}-\dot{\boldsymbol{J}} \dot{\boldsymbol{q}}) \\
& \Upsilon(\dot{\boldsymbol{q}}, \boldsymbol{q})=G^{-T}\left(M(\boldsymbol{q}) \boldsymbol{J}^{-1}\left(\Gamma^{*}-\dot{\boldsymbol{J}} \dot{\boldsymbol{q}}\right)+\boldsymbol{b}(\dot{\boldsymbol{q}}, \boldsymbol{q})+\boldsymbol{g}(\boldsymbol{q})\right)
\end{aligned}
$$

Now, $\Gamma^{*}$ is defined in terms of the actual and desired operational space variables of interest.

$$
\Gamma^{*}(\dot{\boldsymbol{q}}, \boldsymbol{q})=\dot{\boldsymbol{v}}_{\boldsymbol{d}}+k_{v}\left(\boldsymbol{v}_{\boldsymbol{d}}-\boldsymbol{v}\right)+k_{p}\left(\boldsymbol{p}_{\boldsymbol{d}}-\boldsymbol{p}\right)
$$

The angular trajectories require the use of quaternions to prevent singularities. Using the derivation provided in [17], the angular position error $\left(\boldsymbol{\theta}_{\boldsymbol{d}}-\boldsymbol{\theta}\right)$ is found by:

$$
\left(\boldsymbol{\theta}_{\boldsymbol{d}}-\boldsymbol{\theta}\right)=\eta_{d} \boldsymbol{\epsilon}-\eta \boldsymbol{\epsilon}_{\boldsymbol{d}}+\left[\boldsymbol{\epsilon}_{\boldsymbol{d}} \times\right] \boldsymbol{\epsilon}
$$




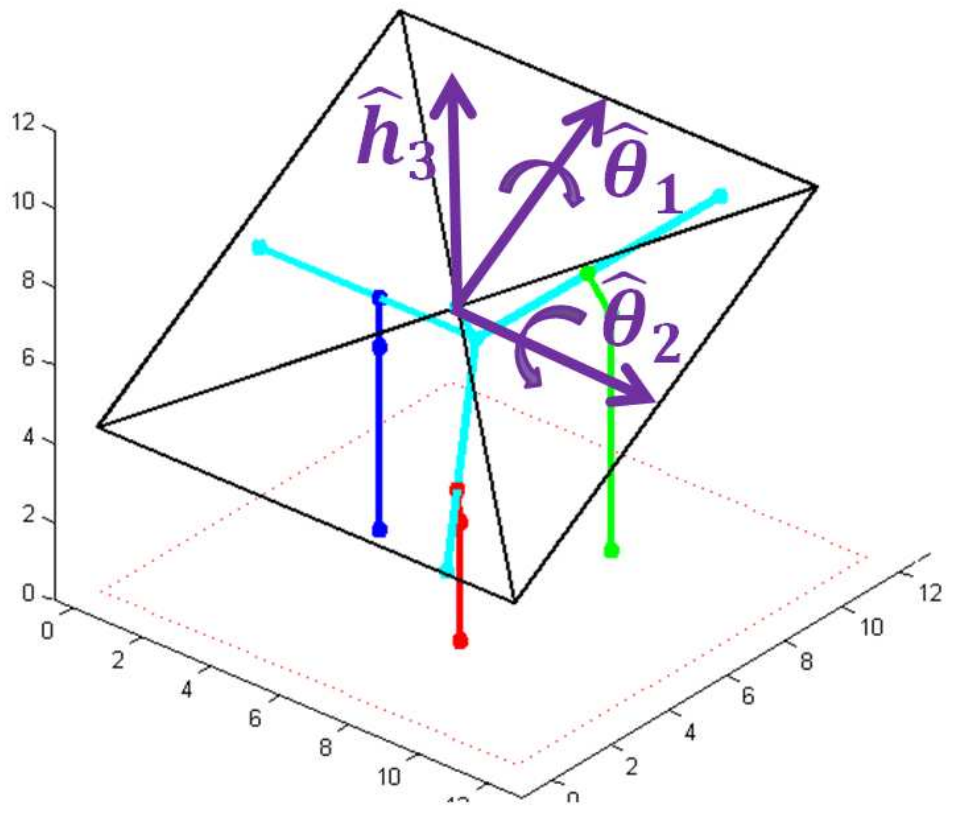

Figure 3.2. Operational space velocities of the mechanism: vertical translation, $\overrightarrow{\boldsymbol{h}}_{\mathbf{3}}$, and two axes of rotation, $\overrightarrow{\boldsymbol{\theta}}_{\mathbf{1}}$ and $\overrightarrow{\boldsymbol{\theta}}_{\mathbf{2}}$.

Where each $\boldsymbol{\theta}$ and $\boldsymbol{\theta}_{\boldsymbol{d}}$ ais defined in quaternion notation (3.13-3.14) and $\left[\boldsymbol{\epsilon}_{\boldsymbol{d}} \times\right]$ is defined in (3.15).

$$
\begin{gathered}
\boldsymbol{\theta}=\left[\eta, \epsilon_{1}, \epsilon_{2}, \epsilon_{3}\right] \\
\boldsymbol{\theta}_{\mathbf{1}}=\left[\cos \left(\theta_{1} / 2\right), \sin \left(\theta_{1} / 2\right), 0,0\right] \\
\boldsymbol{\theta}_{\mathbf{2}}=\left[\cos \left(\theta_{2} / 2\right), 0, \sin \left(\theta_{2} / 2\right), 0\right] \\
{\left[\boldsymbol{\epsilon}_{\boldsymbol{d}} \times\right]=\left[\begin{array}{ccc}
0 & -\epsilon_{3 d} & \epsilon_{2 d} \\
\epsilon_{3 d} & 0 & -\epsilon_{1 d} \\
-\epsilon_{2 d} & \epsilon_{1 d} & 0
\end{array}\right]}
\end{gathered}
$$

To sum up, the actuator torque provided by the three motors is determined by (3.9) and (3.10) to minimize the error between the actual operational space position and velocity of the plate and the desired operational space trajectory of the plate. 
The desired trajectory of each plate on the Smartbed surface is determined in a two-step fashion. First, the desired final position of each plate is calculated based on a requested change in position. For instance, if the bed is changing from a flat surface to a partially inclined surface such as semi-Fowler position, some of the plates will raise and rotate by $30^{\circ}$. Next, the motion of the whole bed is coordinated using trapezoidal velocities. This is a common method for trajectory calculation, and the slow speeds at which the plates move will create very little jerk due to the discontinuous acceleration profile [18]. With trajectory calculation, the linear and rotational velocities increase linearly at a rate of $a_{\max }$ to a maximum velocity $\left(v_{\max }\right)$, remain constant, and then decrease linearly at $-a_{\max }$. Values for $v_{\max }$ and $a_{\max }$ are set according to the maximum motor torque and velocities specified by the manufacturer.

The total time required for the bed to change positions is equal to the time necessary for the "most stressed" threaded rod to reach its final position. The most stressed rod is defined as the rod that has the largest change in position in joint space for a given bed movement. The lengths of travel for all rods are approximated according to the following equations, where the maximum length of travel, $L_{\max }$, is simply the maximum change in position of the three rods $\left(d r_{1}, d r_{2}\right.$, and $\left.d r_{3}\right)$ for all 28 plates.

$$
\begin{aligned}
d r_{1} & =d h_{3}+l_{1} \tan \left(d \theta_{2}\right) \\
d r_{2} & =d h_{3}-l_{4} \tan \left(d \theta_{2}\right)+l_{5} \tan \left(d \theta_{1}\right) \\
d r_{3} & =d h_{3}-l_{4} \tan \left(d \theta_{2}\right)-l_{5} \tan \left(d \theta_{1}\right) \\
L_{\max } & =\max \left(d r_{1}, d r_{2}, d r_{3}\right)_{n} \text { for plates } n \in\{1, \ldots, 28\}
\end{aligned}
$$

The constant $l_{1}$ is the length from rod 1 to the center of the top plate in the $\hat{N}_{1}$ direction when the top plate is horizontal, and $l_{4}$ and $l_{5}$ are the distances from the center of the top plate to rods 2 and 3 in the $\hat{N}_{1}$ and $\hat{N}_{2}$ directions, respectively 3.3 . 


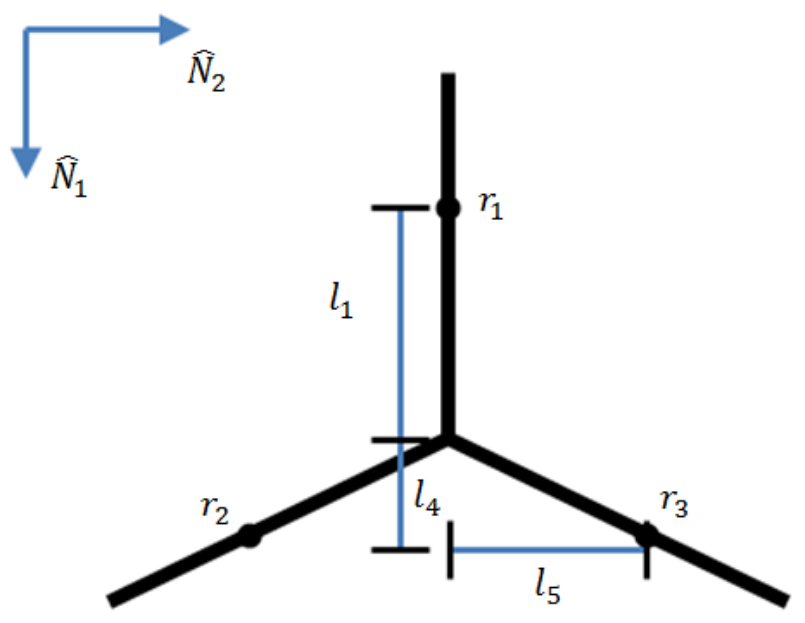

Figure 3.3. Top view of the mechanism schematic, illustrating the definitions of $l_{1}$, $l_{4}$, and $l_{5}$ with respect to the threaded rods $r_{1}, r_{2}$, and $r_{3}$.

$d \theta_{1}, d \theta_{2}$, and $d h_{3}$ are the maximum operational space distances traveled by each plate, and the equations are based on the Jacobian when the plate is perfectly horizontal. The plate is not expected to rotate by large amounts, and any underestimation in the equations is remedied by setting $v_{\max }$ to a value lower than the motors' maximum speed. With $L_{\max }$ known, the total time for the coordinated motion of the bed is calculated as follows.

$$
t_{t o t a l}=\frac{L_{\max } a_{\max }+v_{\max }^{2}}{a_{\max } v_{\max }}
$$

The trajectory of each plate is then set according to a synchronized total time, $t_{\text {total }}$, as well as rise and fall times, $t_{\text {rise }}$ and $t_{\text {fall }}$. The resulting trajectories in operational space for each plate are then calculated as piecewise functions of time:

$$
p(t)= \begin{cases}p_{i}+v_{\text {max }} t^{2} / 2 t_{\text {rise }} & 0 \leq t<t_{\text {rise }} \\ p_{r}+v_{\text {max }}\left(t-t_{\text {rise }}\right) & t_{\text {rise }} \leq t<t_{\text {fall }} \\ p_{\text {final }}-v_{\text {max }}\left(t_{\text {total }}-t\right)^{2} / 2 t_{\text {fall }} & t_{\text {fall }} \leq t \leq t_{\text {total }}\end{cases}
$$

The exact same piecewise equation is used for all three operational space variables, $h_{1}, \theta_{1}$, and $\theta_{2}$. Desired velocity and acceleration functions are found by simply dif- 
ferentiating these functions according to time. Simulations of this control scheme in action are found in chapter 5 . 


\section{CHAPTER 4}

\section{CONTROL SYSTEM APPLICATION}

The control equations derived in the previous chapter are applicable only to an "ideal" mechanism in simulataion because they are based on the following assumptions:

1. Initial values for all 13 joint space positions and at least 3 velocities are known or are measurable before the control system takes over.

2. Exact positions of each rod with respect to an inertial reference point are known or are measurable at all times.

3. Nonlinearities such as friction are not present in the actuation system.

4. The controller has enough memory to house the control program and is powerful enough to perform the necessary calculations and provide torque signals to each motor in real time.

However, in reality these are rarely the case - position data can only be provided where sensors are present, these positions are often only provided as a relative measurement from an encoder, friction and other nonlinearities in actuation are often significant, and laboratory computers have difficulty handling the full complexity of an accurate computed-torque control scheme, let alone in real time. In order to apply the ideal control scheme to the Smartbed hardware, these issues must first be addressed. It is the application of this control system to actual hardware that provides the novelty of this work - whereas computed-torque control equations based on this parallel mechanism have been previously determined, no one has yet taken the steps necessary to effectively apply these equations to the constructed system. 


\subsection{Simplification of the Control Equations}

One issue with the planned control system was that the torque equations developed based on the previously discussed dynamic models are too complex. These equations need tens of thousands of lines to run, and they require too much computing time to be run in real time, even for the relatively slow speed of this mechanism. In order to solve this problem, shorter torque equations are developed based on a simplified model of the mechanism (fig. 4.1).
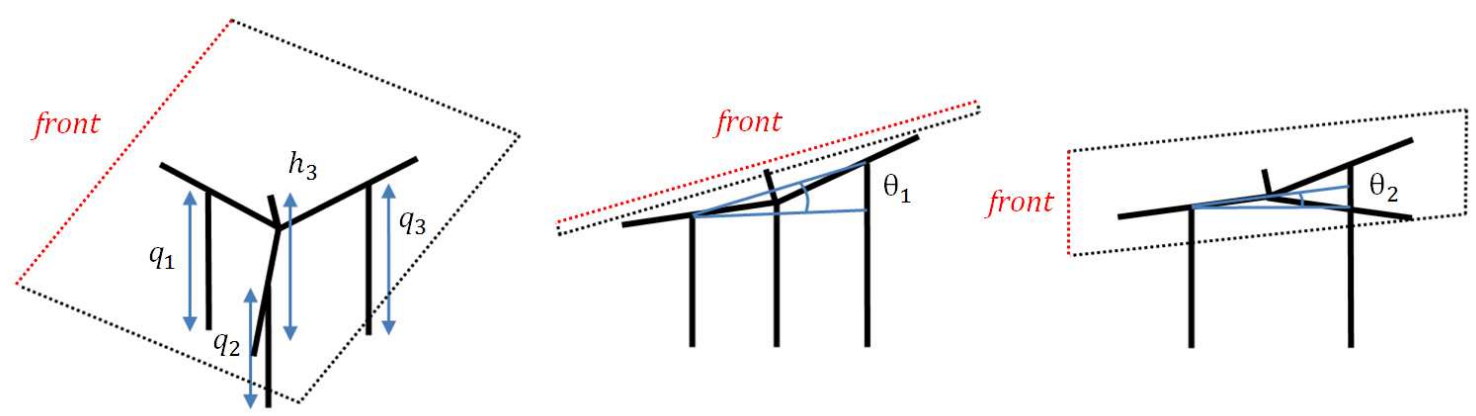

Figure 4.1. Illustration of the simplified mechanism model, relating the operational space variables $\theta_{1}, \theta_{2}$, and $h_{3}$ and the joint space variables, $q_{1}, q_{2}$, and $q_{3}$. The front of the mechanism in each image is labeled for reference.

The simplified model assumes that the three operational space variables, $\theta_{1}, \theta_{2}$, and $h_{3}$ can be approximated by the equations:

$$
\begin{gathered}
\theta_{1}=\tan ^{-1}\left(\left(q_{3}-q_{2}\right) / l_{\text {width }}\right) \\
\theta_{2}=\tan ^{-1}\left(\left(2 q_{1}-q_{1}-q_{2}\right) / l_{\text {long }}\right) \\
h_{3}=\frac{1}{3}\left(q_{1}+q_{2}+q_{3}\right)+l_{\text {top }} \cos \left(\theta_{1}\right) \cos \left(\theta_{2}\right)+l_{\text {joint }}
\end{gathered}
$$

where $l_{\text {length }}$ is the distance between the base of rod $q_{1}$ and the midpoint between $q_{2}$ and $q_{3}, l_{\text {width }}$ is the distance between the bases of rods $q_{2}$ and $q_{3}, l_{\text {top }}$ is the distance between the center of the three-pronged body and the center of the top plate, and 
$l_{\text {joint }}$ is the length of the three joints on top of $q_{1}, q_{2}$, and $q_{3}$. The $\theta_{1}$ approximation is based solely on the difference between $q_{2}$ and $q_{3}$, and the $\theta_{2}$ approximation is based solely on the difference between $q_{1}$ and the average of $q_{2}$ and $q_{3}$. The approximated $h_{3}$ is then equal to the average of $q_{1}, q_{2}$, and $q_{3}$ with the addition of the the length of one joint and the height of the top plate above the three-pronged structure, adjusted based on its angle.

These equations are important because they are only based on three quantities: $q_{1}, q_{2}$, and $q_{3}$. These are the only measurable quantities for the mechanism, and the control system must be able to function with only these values as inputs.

When the plate is perfectly horizontal, these equations exactly match the accurate model derived in chapter 3 . However, as $\theta_{1}$ and $\theta_{2}$ move away from $0^{\circ}$ to their operational space limits of $\pm 30^{\circ}$, the accuracy of each equation decreases. In order to counter this, best-fit curves for the error of each equation are determined. Since the kinematics of this parallel mechanism are extremely complex, appropriate polynomial fit curves for each operational space variable are determined through trial and error.

Fig. 4.2 shows the difference between the value for $\theta_{1}$ determined by the original, "accurate" model's kinematic equations and the value for $\theta_{1}$ determined by the new, simplified model's kinematic equations. The error in $\theta_{1}$ is on the $y$ axis, and the value of $\theta_{1}$ predicted by the accurate model is on the $x$ axis. This plot is useful because it shows the ability of the simplified model to determine close approximate values of the operational space variables in terms of the measurable quantities. The graph also shows where the maximum error occurs over the full domain of $\theta_{1}\left( \pm 30^{\circ}\right)$. The further the red dotted line is from the blue horizontal line, the worse the approximation.

As seen by this plot, the error in $\theta_{1}$ is negligible - the approximated and actual values differ by less than $.0004^{\circ}$. This makes sense, as the mechanism is symmetric 


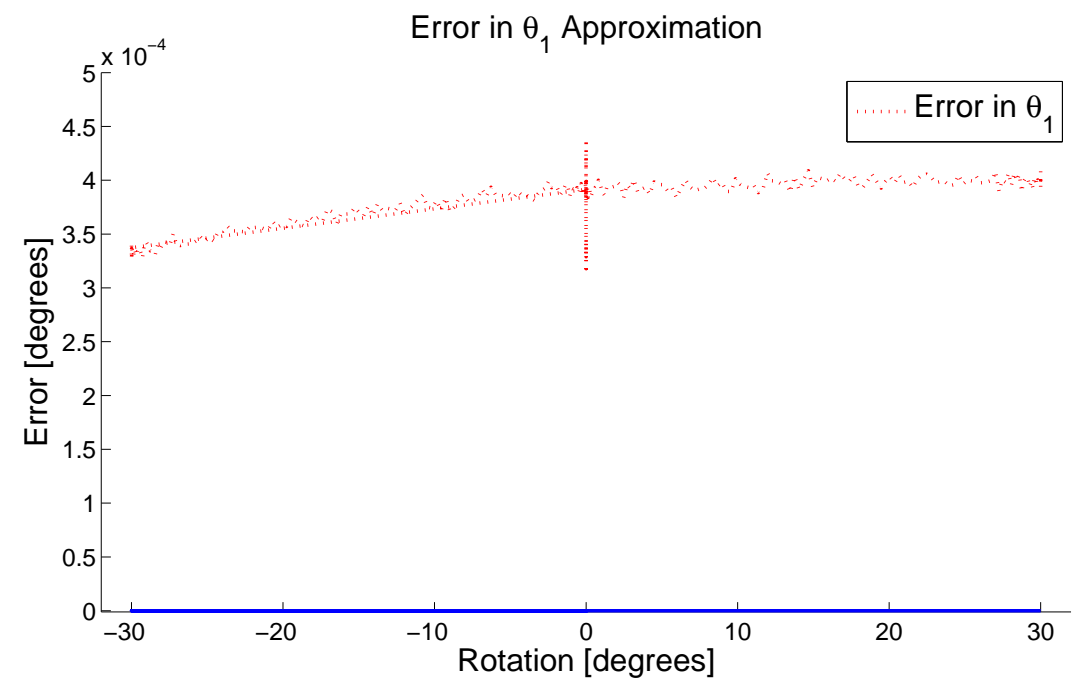

Figure 4.2. Error in approximated $\theta_{1}$ value over the full operational domain of $\theta_{1}$. The solid line at the bottom represents where there is exactly zero error.

about the plane defined by $\hat{N}_{1} \times \hat{N}_{2}$. Based on this information, (4.1) is shown to be a very accurate approximation of the value for $\theta_{1}$.

The error between the accurate and simplified $\theta_{2}$ equations is much more significant and includes prominent quadratic and linear terms. The coefficients for these error terms are first approximated using a second-order polynomial fit and then cancelled out as best as possible by subtracting them from the initial approximation. In addition, the error of $\theta_{2}$ increases as $\theta_{1}$ approches $\pm 30^{\circ}$. Because of this, a separate polynomial fit is determined for $\theta_{2}$ based on $\theta_{1}$ and subtracted from (4.2).

The same process of polynomical curve-fitting, determining term coefficients, and subtracting the terms is applied to the error in $h_{3}$ based on $\theta_{1}$ and $\theta_{2}$, resulting in the following equations. $l_{\text {width }}$ is the distance between rods 2 and $3, l_{\text {length }}$ is the distance between rod 1 and the center point between rods 2 and $3, l_{\text {top }}$ is the height of the plate above the three-pronged structure that connects the three rods, and $l_{\text {joint }}$ is the vertical height of each joint connecting the rods and the three-pronged structure. 
The variable temp is simply a temporary variable included to simplify the equation for $\theta_{2}$.

$$
\begin{gathered}
\theta_{1}=\tan ^{-1}\left(\left(q_{3}-q_{2}\right) / l_{\text {width }}\right) \\
\text { temp }=\tan ^{-1}\left(\left(2 q_{1}-q_{1}-q_{2}\right) / l_{\text {length }}\right) \\
\theta_{2}=.0944(\text { temp })^{2}+1.0042(\text { temp })-.11 \theta_{1}^{2} \\
h_{3}=\frac{1}{3}\left(q_{1}+q_{2}+q_{3}\right)+l_{\text {top }} \cos \left(\theta_{1}\right) \cos \left(\theta_{2}\right)+1.028 l_{\text {joint }}+.33 \theta_{1}^{2}-.22 \theta_{2}
\end{gathered}
$$

The maximum resulting error in $\theta_{1}, \theta_{2}$, and $h_{3}$ based on these approximations is $.0004^{\circ}, .074^{\circ}$, and $1.5 \mathrm{~mm}$, respectively (Fig. 4.2, 4.3a-b). These values are considered negligible compared to the measurement error present in $q_{1}, q_{2}$, and $q_{3}$ and the level of accuracy required to manipulate a human patient.
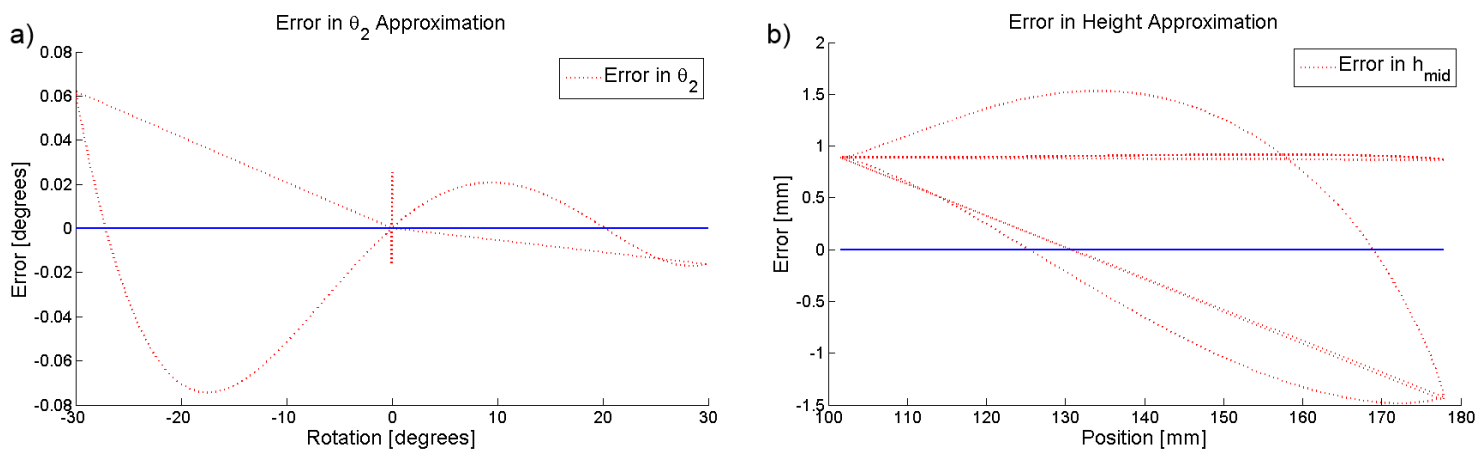

Figure 4.3. a) Error in approximated $\theta_{2}$ value over the full operational domain of $\theta_{2}$. b) Error in approximated $h_{3}\left(h_{\text {mid }}\right)$ value over the full operational domain of $h_{3}$. The blue lines represent the ideal case where there is exactly zero error.

The resulting kinematic equations are short, accurate, based only on measureable variables, and require very little processing power to perform in real time. Thus, they can be applied to the microcontroller for this system. 


\subsection{Consideration of Actuator Dynamics}

The actuation of the three rods is provided by three motors placed on the base plate of the mechanism. Torque from the motors is rotated $90^{\circ}$ by a worm gear and then transformed into vertical force by a leadscrew and nut. The ideal dynamic model described in the previous chapter considers the inertias of the motor, shaft coupling (sc), worm, worm gear (wg), and leadscrew (ls) in its calculations, determined according to (4.8).

$$
I_{\text {total }}=I_{\text {motor }}+I_{s c}+I_{w o r m}+R_{1}^{2}\left(I_{w g}+I_{l s}\right)=5.1 \mathrm{kgmm}^{2}
$$

$R_{1}$ is equal to $1 / 50$, which is the gear ratio of the worm gear. The effect of this total gear train inertia on the plate is enhanced due to the effect of reflected inertia by multiplying by the square of the total gear ratio of the worm gear and leadscrew, $R^{2}$. Taking this into account, the effective gear train inertia is $710 \mathrm{kgmm}^{2}$, which is two orders of magnitude less than the inertia of the top plate $\left(I_{x x}=I_{y y}=14,833 \mathrm{kgmm}^{2}\right.$, $I_{z z}=28,909 \mathrm{kgmm}^{2}$ ). This means that the inertia of the gear train has a very small effect on the overall system, and as a result is ignored in the final simplified control equations.

In addition, the Coriolis and centrifugal effects determined in simulation are very small for this mechanism since it moves so slowly. These values are many orders of magnitude smaller than the effect of gravity and inertia and can therefore also be ignored in the final simplified control equations.

Finally, the effect of friction in the leadscrew must be considered. Because of the low lead angle for the threaded rod, the weight applied to the top plate is always supported by the base plate through the leadscrew nut and a rotational bearing - the gear train and motor are never responsible for the weight of the plate, mattress, and patient. Therefore, in order to provide movement, the motor only needs to overcome 
the friction forces between the leadscrew and the leadscrew nut. This is analogous to moving a block placed on an inclined plane, since the screw would form a plane if "unwrapped" from the rod (Fig. 4.4). The angle of friction, $\phi$, is the minimum angle required for the block (analogous to the threaded rod) to overcome static friction and release down the inclined plane without help from an applied force (analogous to the motor torque). Since the lead angle, $\lambda$, is less than $\phi$, the motor torque must only overcome the friction force in order to actuate the threaded rod.

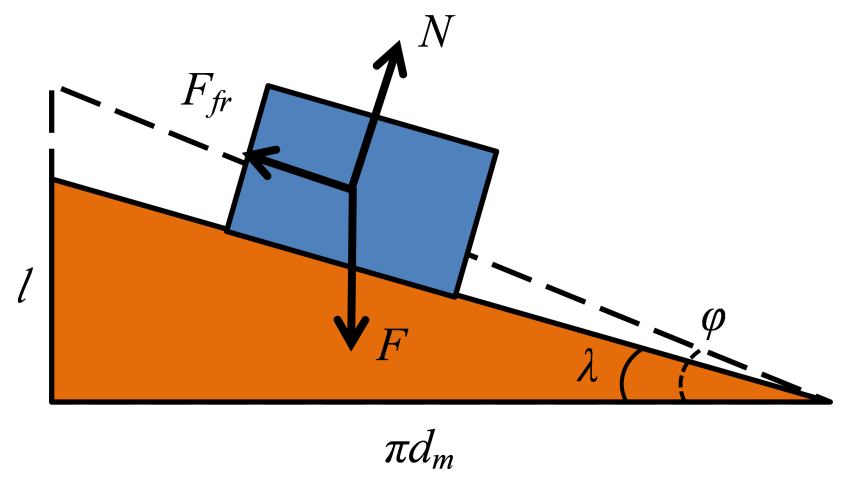

Figure 4.4. Inclined plane analogous to the incline of the leadscrew's thread. $l$ is the lead length (the vertical distance traveled when the thread completes one rotation) and $\pi d_{m}$ is the mean circumference of the thread for one rotation.

As a result of this, it is more accurate to consider the actuation provided by the overall torque control equations through the effect of friction between the leadscrew threads. The following two equations are used to determine the transfer of torque through a leadscrew being raised and lowered and are based on the applied force $(F)$, mean leadscrew diameter $\left(d_{m}\right)$, dynamic coefficient of friction $\left(\mu_{d}\right)$, lead angle $(\lambda)$, and thread angle $(\alpha)$.

$$
\begin{aligned}
\tau_{\text {raise }} & =\frac{F d_{m}}{2}\left(\frac{\mu_{d} \sec (\alpha)+\tan (\lambda)}{1-\mu_{d} \sec (\alpha) \tan (\lambda)}\right) \\
\tau_{\text {lower }} & =\frac{F d_{m}}{2}\left(\frac{\mu_{d} \sec (\alpha)+\tan (\lambda)}{1+\mu_{d} \sec (\alpha) \tan (\lambda)}\right)
\end{aligned}
$$


Given the leadscrew parameters $d_{m}=15.875 \mathrm{~mm}, \mu_{d} \simeq .10, \alpha=29^{\circ}$ and $\lambda=4.9^{\circ}$, both equations yield force-torque relations of $\tau=.0016 F$. The lack of discrepancy between raising and lowering is due to the small values for both $\mu_{d}$ and $\lambda$, effectively making the denominator of both equations equal to 1 . This torqueforce relationship is different than the previously determined linear velocity-angular velocity gear ratio for the leadscrew because of the effect of friction. To account for this in the model, the torque is multiplied by a lead screw efficiency term, $\eta_{l s}$. This is multiplied by the manufacturer's motor efficiency to create an overall actuating system efficiency.

$$
\begin{aligned}
\eta_{l s} & =\frac{\tan (\lambda)}{\tan (\phi+\lambda)}=\frac{\tan \left(4.9^{\circ}\right)}{\tan \left(29^{\circ}+4.9^{\circ}\right)}=.34 \\
\eta_{\text {total }} & =\eta_{l s} \eta_{\text {motor }}=\quad .34(.80)=.27
\end{aligned}
$$

Finally, the direction of friction is taken into account by multiplying the previous equations by the function $\operatorname{sign}\left(\dot{q}_{n}\right)$, where $n$ refers to threaded rod 1,2 , or 3 , since friction always opposes motion. These yields a final torque equation of:

$$
\boldsymbol{\Upsilon}(\dot{\boldsymbol{q}}, \boldsymbol{q})=\eta_{t o t a l} G^{-T}\left(M(\boldsymbol{q}) \boldsymbol{J}^{-\mathbf{1}}\left(\boldsymbol{\Gamma}^{*}-\dot{\boldsymbol{J}} \dot{\boldsymbol{q}}\right)+\boldsymbol{g}(\boldsymbol{q})\right) * \operatorname{sign}\left(\dot{q}_{n}\right)
$$

with $\Gamma^{*}$ defined by $\boldsymbol{\Gamma}^{*}(\dot{\boldsymbol{q}}, \boldsymbol{q})=\dot{\boldsymbol{v}}_{\boldsymbol{d}}+k_{v}\left(\boldsymbol{v}_{\boldsymbol{d}}-\boldsymbol{v}\right)+k_{p}\left(\boldsymbol{p}_{\boldsymbol{d}}-\boldsymbol{p}\right)$, just as before.

In summary, the dynamics of the actuating system are taken into account by combining the gravity and inertia terms in the equations of motion, multiplying them by an efficiency coefficient of 0.27 , and then finally selecting the sign based on their direction of motion. In addition, the tiny reflected inertia of the motor and gear train is ignored, which shortens and simplifies the final control equations. 


\section{CHAPTER 5}

\section{RESULTS AND EXPERIMENTAL TESTING}

The results of this work can be summed up into two aspects: a viable control program and a working plate prototype. The control program is described first, followed by an outline of the measures taken to make the physical mechanism operational.

\subsection{Control Program}

The mechanism is controlled by a Texas Instruments (TI) TMX320C28345ZHH microcontroller placed on a prototyping board plugged into a docking station. The microcontroller is run by a program which includes the basic program setup, input/output pin configuration, and all of the code required to control the mechanism.

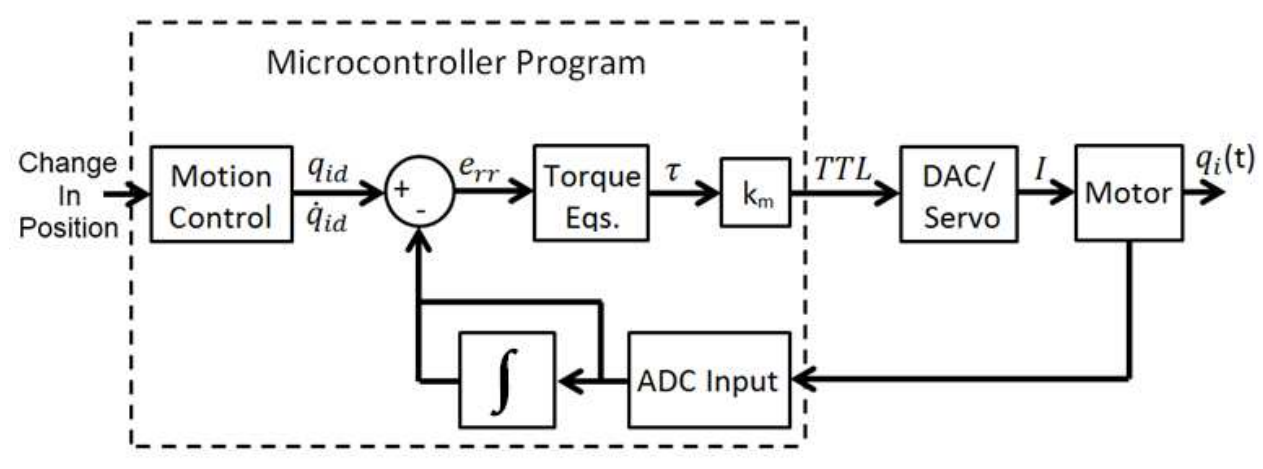

Figure 5.1. Control diagram for the mechanical actuators in each tile.

The control functions of the microcontroller program are set up as follows: first, a desired bed shape is inputted into the microcontroller, indicating which posi- 
tion (right-side lateral, left-side lateral, flat, or semi-Fowler's) the the patient should be changed to (Fig. 5.1). Next, the desired positions of each tile are found to create the desired bed shape. Trajectory equations then determine desired operational space positions over time, comparing this to the tile's actual position and velocity to generate control errors. Using the torque equations determined in chapters 3 and 4 , digital signals are produced and sent to a digital to analog converter (DAC) based on these errors. The DAC outputs analog signals to three servoamplifiers, one each for the three motors. Each motor then produces a proportional torque, and the motor's rotational velocity is read by a digital encoder. This encoder signal is combined with a known positional set-point when the mechanism is fully down to produce the position and velocity feedback, read by the microcontroller through an analog to digital converter input (ADC Input).

The program is first tested by comparing the simplified control program to the accurate model in Matlab. In order to accomplish this, the accurate control equations in a numerical simulation of the mechanism are replaced by the simplified control equations to test their effectiveness. If the simplified control equations are able to make the plate effectively follow the desired trajectory, then the program is considered successful.

Figure 5.2a-b show the results of one test performed by the simplified equations. The dotted lines represent the desired trajectory of the plate in operational space, and the solid lines represent the mechanism's actual trajectory. It is easily apparent that the control equations are effective, yielding negligible offsets in position and velocity as well as a non-existent overshoot at all three non-smooth corners in the velocity trajectory. The only visible error is a slight delay in angular acceleration, but this should not affect the quality of the position change. 

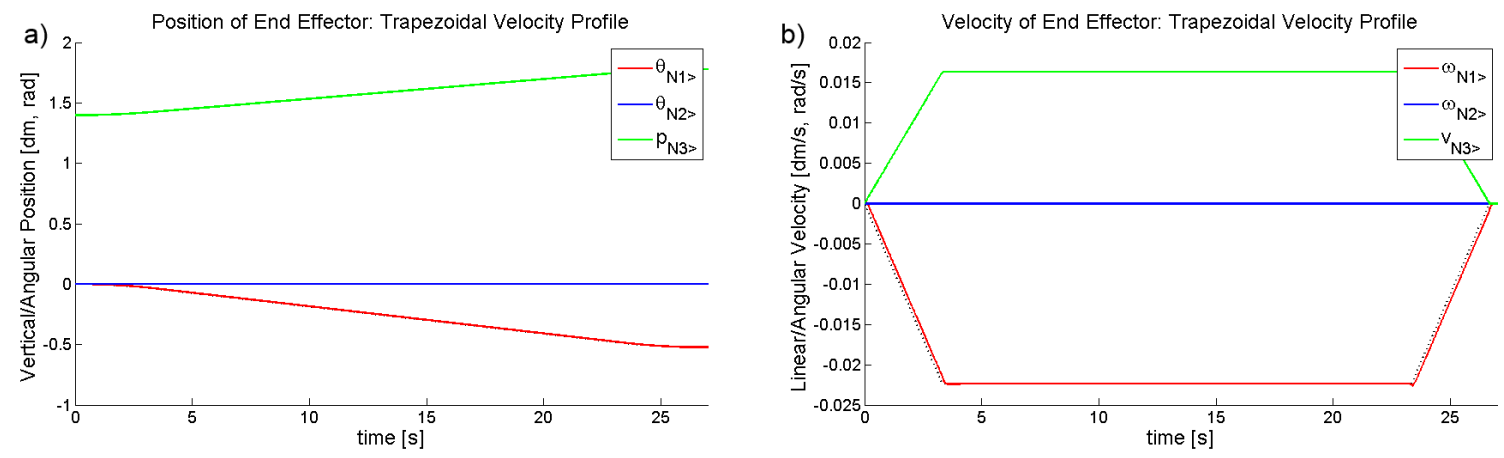

Figure 5.2. Desired trapezoidal trajectories shown as dotted lines are followed closely by the actual mechanism positions (a) and velocities (b) in simulation.

The torque required to create these trajectory profiles is shown below in Fig. 5.3. The motors are found to use approximately $2 \mathrm{~A}$ of current to move the plate, which relates to a maximum motor torque of $41 \mathrm{mNm}$, close to the simulated torque's predicted maximum of about $40 \mathrm{mNm}$.

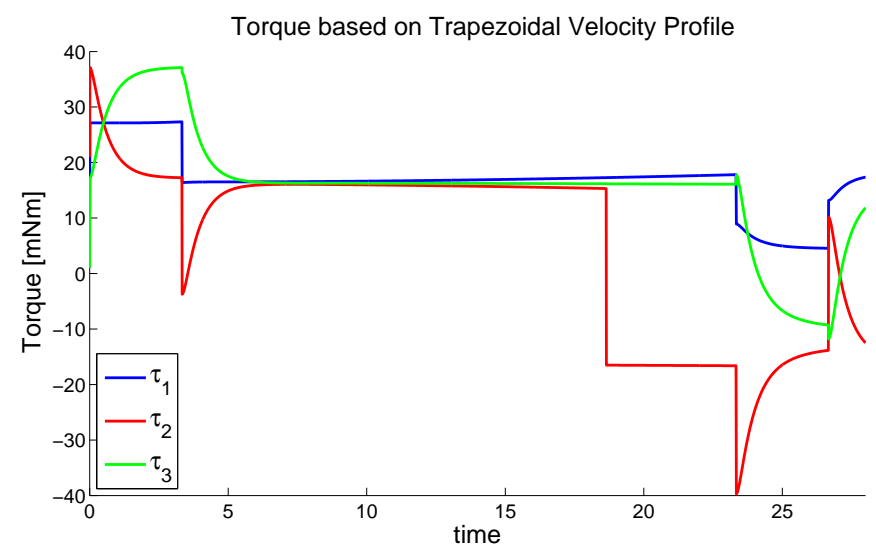

Figure 5.3. Motor torques required to create the previously illustrated operational space trajectories. The spikes represent where the discontinuities in desired acceleration exist at $t(0), t_{\text {rise }}, t_{\text {fall }}$, and $t_{\text {total }}$. In addition, at $t \approx 18$, the sign of $\tau_{2}$ changing from + to - is apparent.

The plate also requires no torque to stay at a constant height due to the $\operatorname{sign}(X)$ function applied to the torque because of friction. These two extreme values agree 
with the values shown in the simulation, and the slopes of each line appear to be correct. So, the torque equations appear to be accurate.

\subsection{Mechanism Hardware}

Before the research communicated in this work began, part of the mechanism had been already manufactured. The top plate, joints, threaded rods, leadscrew, worm gear, and base plate were already constructed and assembled. However, the device did not work - in addition to not have a viable control program, the previously selected motors ran extremely slow, taking minutes to perform significant changes in plate position. In order to fix this problem, new motors have been selected, purchased, and implemented on the mechanism (Fig 5.4).

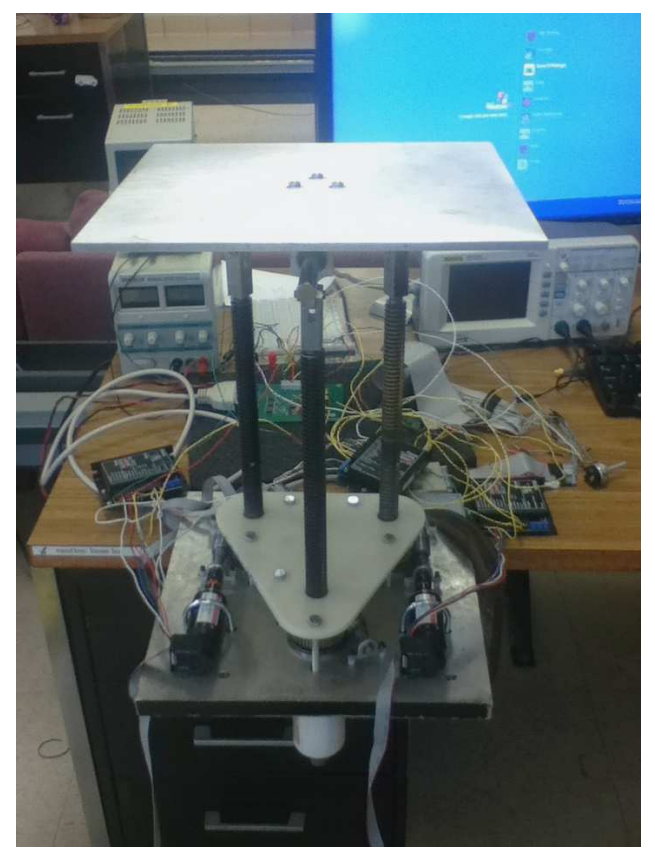

Figure 5.4. One mechanism, fully constructed. This image includes the new motors, shaft couplings, a hard plastic bracket, and all necessary electronics. 
Motor selection requires the consideration of three constraints with respect to torque, speed and power: the maximum torque-speed curve, power constraint, and thermal constraint, each of which is provided by the manufacturer. Motor cost and availability are also considered, as well as the cost and availability of auxiliary components that are compatible with each motor (encoder, gear box, and servoamplifier). After considering all of these aspects, the motor combination from Maxon selected for this application is the EC 32, 80W motor (\#118889), Maxon DEC 50V/5A 1-Q-EC Amplifier (\#230572), and HEDL 5540 encoder with line driver (\#110514).

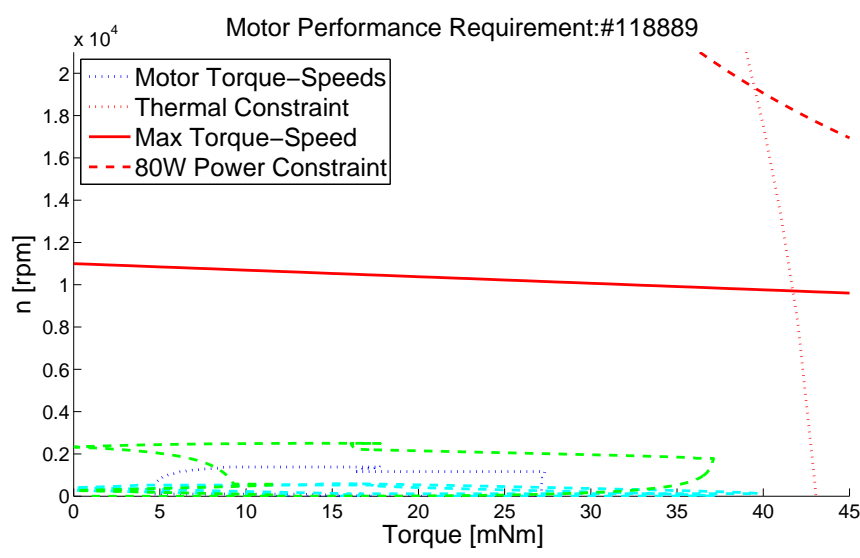

Figure 5.5. Torque-speed curves shown against the working range of the motor. All curves are well within the thermal, power, and motor torque-speed limits.

According to Fig. 5.5, this combination can provide adequate torque to actuate the threaded rods for all possible movements in the plate's operational space domain. The blue and green curves show the motor torque-speeds required by all three motors while moving vertically and rotating about both the $\hat{N}_{1}$ and $\hat{N}_{2}$ axes. Since all of these curves stay below and to the left of the three constraints set by the manufacturer in the torque-speed domain, the motors will not require more torque or more speed than they can provide. 
One very important aspect of this setup is that no gear box is required to increase the torque of the motor-removing the 1:53 ratio gear box on the previous motors increases the speed of the mechanism by 53 times, reducing necessary for the plates to change position from minutes to the order of seconds. For example, as stated in chapter 2, each rod can fully extend in approximately 30 seconds, a motion that would have taken 25 minutes with the previous motor.

In order to incorporate the motors into the original design, a few changes have been made to the hardware. New shaft couplings are in place to account for the larger shaft diameter, and spacers are placed underneath the leadscrew to raise it to the height of the new motor shaft (Fig. 5.6a). The leadscrews are also placed under custom connectors designed to keep the rods from dislocating from the base plate (Fig. 5.6b). These connectors are rapid prototype parts and utilize rotational bearings to allow for free rotation of the leadscrew nuts while still applying downward pressure to keep the leadscrew in place (Fig. 5.6c).

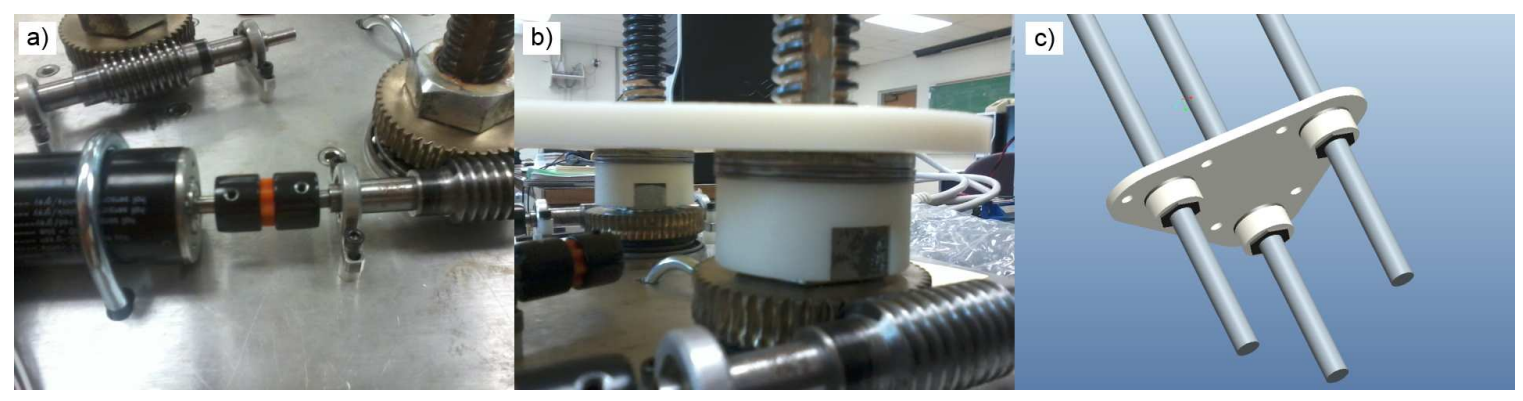

Figure 5.6. a) Motor, shaft coupling, worm, worm gear, and leadscrew. b) The offwhite bracket locks the leadscrew and threaded rods in place through a thick washer, a rotational bearing, a thin washer, and a rapid prototype connector stuck directly on the leadscrew nut. c) The 3-D model used to create the rapid-prototype parts.

Testing of the new hardware setup is conducted using an open-loop user input signal provided by a potentiometer. The voltage output of the potentiometer is 
between $0 \mathrm{~V}$ and $5 \mathrm{~V}$, sent into the microcontroller through an analog input on the TI docking station. This control signal is divided into three regions: clockwise movement, brake (no movement), and counter-clockwise movement, allowing the plate to be raised, lowered, and stopped by adjusting the position of the potentiometer knob (Fig. 5.7). This utilizes speed control as opposed to the actual closed-loop program which is based on torque control.

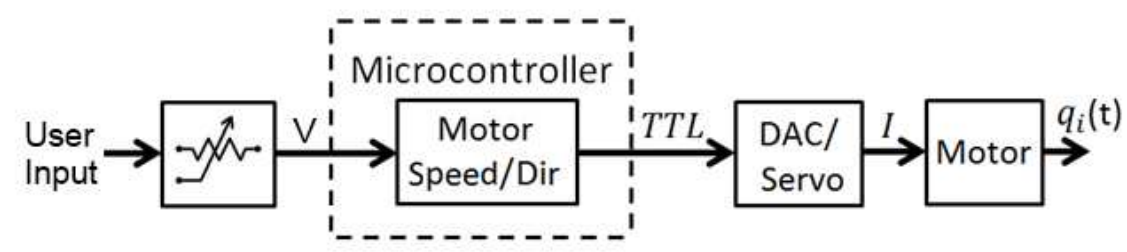

Figure 5.7. Control diagram for the mechanical actuators in each tile.

While performing this test, all three rods were easily raised and lowered quickly without damage to the hardware or electronics. All components stayed in place, and the mechanism's gears and joints never locked. Based on this test the quality and effectiveness of the hardware is confirmed, and the hardware portion of this project is considered successful.

The final step for testing and verification requires combining the closed-loop, simplified control program with the working hardware setup. Unfortunately, this has not been completed - closed loop control requires that the encoder signal be sent to and interpreted by the microcontroller, and this process has not been implemented. The encoder requires a specific integrated cicuit (IC) in order to interpret the encoder data, which would then have to provide either a proportional digital or analog signal to the microcontroller. In addition, a more complicated user input system would have to fully test the capability of the designed system. This would include a way to 
rotate between the lateral, flat, and semi-Fowler positions previously described in this report in addition to the potentiometer input that raises and lowers the top plate. Finally, a method of determining absolute position of each rod would be required at program startup. With an accurate initial absolute position, the encoder could take over position monitoring without issue. If these three components were implimented, a simple test of positon changing would show that the single plate mechanism works as intended, and the prototype is therefore complete. 


\section{CHAPTER 6}

\section{CONCLUSION}

This work details the design, modeling, simulation, and open loop testing of the actuating mechanism for a smart hospital bed manufactured to prevent the development of bed sores. The steps taken to augment an unfinished, immobile prototype of the mechanism with the use of theoretical models and practical analysis are illustrated, and the resulting mechanism control program and hardware are provided.

First, a dynamic model of the system in created in order to select the motors and related components based on torque-speed contraints for each motor provided by the manufacturer. Next, the model is used to determine control equations capable of making the plate follow desired operational space trajectories. These equations are then simplified, taking into account the speed, memory, and processing capacity of the microcontroller in order to make them actually usable. After altering the equations further to better model the actuating system, the capability of these simplified control equations is verified in simulation. Finally, an open-loop test of the mechanism hardware and some aspects of the microcontroller code is conducted to qualitatively assess the functionality of the hardware. With the conclusion of these tests, all aspects of the mechanism and control system designs are considered successful, with only the encoder feedback and closed-loop control feedback equations left unverified on the prototype.

The novelty of this work lies in the successful application of an ideal control system developed in simulation to an actual mechanical device. The use of polynomial curve fitting techniques, trapezoidal velocity propagation, and leadscrew dynamics in 
the model transform the dynamic modeling and computed torque equations from a simple academic exercise into a viable, applicable control system. Further work on this project would be focused on the implementation of position sensing, more complex user input, and closed-loop feedback on the mechanism. In addition, modeling and design of the pneumatic system is necessary in order to account for the air bladders on top of each plate. Finally, a control system would have to be developed to combine the 84 rods and 28 air bladders into one coordinated patient-manipulating Smartbed system. With this complete, the bed could be built in its entirety and move on to testing with real patients. 
APPENDIX A

EQUIPMENT LIST 
This appendix includes tables listing all major hardware and software components utilized in the development and testing of the Smartbed mechanism prototype. Part identification numbers and manufacturers are provided where possible.

Table A.1. Hardware Components

\begin{tabular}{|c|c|c|}
\hline Part Name & Part Number & Manufacturer \\
\hline Microcontroller & TMX320C28345ZHH & Texas Instruments \\
\hline$\mu$ C Control Card & Delfino C2834x DIM100 & Texas Instruments \\
\hline Docking Station & BH28xxx & Texas Instruments \\
\hline DAC/Multiplexer & EVAL-AD7839/41EBZ & Analog Devices \\
\hline SMB Plug for DAC/MPX Output & $415-0001-012$ & Emerson Network Power \\
\hline Power Supply & 382202 & Extech Instruments \\
\hline Power Supply & PS3003U & HQ Power \\
\hline Servoamplifier & 230572 & Maxon Motor \\
\hline DC Electric Motor & 118889 & Maxon Motor \\
\hline Digital Encoder & 110514 & Maxon Motor \\
\hline Sleeved Set Screw Shaft Coupling & CPF 16-5-5 & Misumi USA \\
\hline
\end{tabular}

Table A.2. Software Programs

\begin{tabular}{|c|c|c|}
\hline Software Name & Version Number & Developer \\
\hline Matlab & R2012a & MathWorks \\
\hline Autolev & Professional Version 4.1 & OnLine Dynamics, Inc. \\
\hline Code Composer Studio & 4.2 .3 & Texas Instruments \\
\hline
\end{tabular}




\section{REFERENCES}

[1] B. Braden and N. Bergstrom, "Braden Scale - For Predicting Pressure Sore Risk," http://www.in.gov/bradeisdh/files/Braden_Scale.pdf, 1988, [Online; accessed 31-January-2013].

[2] NPUAP, "Pressure Ulcer Prevention Points," http://www.npuap.org/ wp-content/uploads/2012/03/PU_Prev_Points.pdf, Washington DC, 2007, [Online; accessed 31-January-2013].

[3] N. Cullum, J. Deeks, A. Fletcher, T. Sheldon, and F. Song, "Preventing and Treating Pressure Sores," Quality in Health Care, vol. 2, no. 1, pp. 289-297, 1995.

[4] E. Jaul, "Assessment and Management of Pressure Ulcers in the Elderly," Drugs and Aging, vol. 27, no. 4, pp. 311-325, 2010.

[5] D. Gude, "Managing Pressure Ulcers-What is new?" Journal of Mid-Life Health, vol. 2, no. 2, pp. 91-92, 2011.

[6] J. Agostini, D. Baker, and S. Bogardus, Making Health Care Safer: A Critical Analysis of Patient Safety Practices. Rockville, MD: Agency for Healthcare Research and Quality, 2001, Chapter 27. Prevention of Pressure Ulcers in Older Patients.

[7] Y. Jan and M. Brienza, "Technology for Pressure Ulcer Prevention," Topics in Spinal Chord Injury Rehabilitation, vol. 11, no. 4, pp. 30-41, 2006.

[8] C. Lyder, J. Preston, J. Grady, J. Scinto, R. Allman, N. Bergstrom, and G. Rodeheaver, "Quality of Care for Hospitalized Medicare Patients at Risk for Pressure Ulcers," Archives of Internal Medicine, vol. 161, no. 12, pp. 1549-1554, 2001. 
[9] S. Rich, D. Margolis, M. Shardell, W. Hawkes, R. Miller, S. Amr, and M. Baumgarten, "Frequent Manual Repositioning and Incidence of Pressure Ulcers Among Bed-Bound Elderly Hip Fracture Patients," Wound Repair and Regeneration, vol. 19, pp. 10-18, 2011.

[10] S. Rithalia and L. Kenney, "Hospital Bed mattresses: an Overview of Technical Aspects," Journal of Medical Engineering and Technology, vol. 24, no. 1, pp. $32-39,2000$.

[11] S. Rithalia and M. Gonsalkorale, "Assessment of Alternating Air Mattresses Using a TimeBased Interface Pressure Threshold Technique," Rehabilitation Research and Development, vol. 35, no. 2, pp. 225-230, 1998.

[12] S. Rithalia, "Assessment of Patient Support Surfaces: Principle, Practice, and Limitations," Journal of Medical Engineering and Technology, vol. 29, no. 4, pp. 163-169, 2005.

[13] K. Vanderwee, M. Grypdonck, and T. Defloor, "Effectiveness of an Alternating Pressure Air Mattresses for the Prevention of Pressure Ulcers," Age and Ageing, vol. 34, pp. 261-267, 2005.

[14] D. Bain, Evaluation of Nightingale ProAxis Plus Bed with Regard to Dynamic Pressure Attributes, Duncan Bain Consulting, Kings Langley, 2006, [Online; accessed 31-January-2013].

[15] M. More and C. VanGlider, High Yields Result from a Pressure Ulcer Prevention Program, Hill Rom Services, Inc., Yuma, 2006, [Online; accessed 31-January2013].

[16] M. Twiste and S. Rithalia, "Measurement System for the Evaluation of Alternating Pressure Redistribution Mattresses Using Pressure Relief Index and Tissue Perfusion- A Preliminary Study," Wound Practice and Research, vol. 16, no. 4, pp. 192-198, 2008. 
[17] J. Nakanishi, R. Cory, M. Mistry, J. Peters, and S. Schaal, "Operational Space Control: A Theoretical and Empirical Comparison," The International Journal of Robotics Research, vol. 27, no. 6, pp. 737-757, 2008.

[18] J. Craig, Introduction to Robotics: Mechanics and Control, 3rd ed. Upper Saddle River: Pearson Education International, 2005. 


\section{BIOGRAPHICAL STATEMENT}

Zachary G. Brush graduated from Edmond North High School in Edmond, OK, in 2007. He received his Bachelor's Degree in Engineering Science from Trinity University in San Antonio, TX in 2011 and his Master's Degree in Mechanical Engineering from the University of Texas at Arlington in 2013. He worked for the Robotics, Biomechanics, and Dynamic Systems Laboratory for his advisor, Dr. Alan Bowling, while completing his thesis research. 